\title{
ELECTRON-ION STRUCTURE FACTORS AND THE GENERAL ACCURACY OF LINEAR RESPONSE
}

\author{
A.A. Louis \\ University Chemical Laboratories, Lensfield Rd, Cambridge CB2 1EW, UK \\ N.W. Ashcroft \\ Cornell Center for Materials Research, and Laboratory of Atomic and Solid State Physics, \\ Cornell University, Ithaca, NY 14853-2501
}

(October 19, 2018)

\begin{abstract}
We show that electron-ion structure factors in fluid metallic systems can be well understood from an application of linear response in the electron system, combined with hard-sphere like correlation for the ionic component. In particular, we predict that electron-ion structure factors fall into two general classes, one for high $(Z>3)$ and one for low $(Z \leq 2)$ valence metals, and make suggestions for experiments to test these ideas. In addition, we show how the general success of electronic linear response for most metallic systems stems in part from an intrinsic interference between atomic and electronic length scales which weakens the nonlinear response. The main exception to this is metallic hydrogen.
\end{abstract}

\section{INTRODUCTION}

At a near fundamental level, liquid metals are complex binary fluids consisting of ions in a sea of conduction electrons, their physical properties linked to the three corresponding 
correlation functions: $S_{I I}(k), S_{e I}(k)$, and $S_{e e}(k)$ [1]. We present a simple and evidently accurate analytic scheme to calculate electron-ion structure factors $\left(S_{e I}(k)\right)$ by combining a hard-sphere approximation for the ionic structure with a simple linear response theory for the electrons. These structure factors are now in principle accessible experimentally through recent advances in both neutron and x-ray scattering techniques. Another route to effective electron-ion interactions therefore opens, but now through the fluid state.

We also address the evident success of the linear approximation by studying a related problem, the density, $\rho^{\text {ind }}(\overrightarrow{\mathbf{k}})$, of an initially uniform electron gas induced by an embedded pseudo-potential, $v^{p s}(\overrightarrow{\mathbf{k}})$. By comparing linear and second order response to full (KohnSham [2]) non-linear response, we show that even though $v^{p s}(\overrightarrow{\mathbf{k}})$ is not necessarily a small perturbation, the consequent response series converges term by term. The non-linear terms are significantly reduced by an interference between atomic and electronic length scales for most metals, the main exception to this being hydrogen.

\section{DISCUSSION}

\section{A. Electron-ion correlation functions}

The electron-ion-structure factor can be written as [1]:

$$
S_{e I}(k)=\frac{1}{\sqrt{N_{e} N_{I}}}<\hat{\rho}_{e}^{(1)}(\overrightarrow{\mathbf{k}}) \hat{\rho}_{I}^{(1)}(\overrightarrow{\mathbf{k}})>=\frac{n(k)}{\sqrt{Z}} S_{I I}(k),
$$

where $\hat{\rho}_{j}^{(1)}(k)$ is the Fourier transform of the one-particle density operator of component $j$, $S_{I I}(k)$ is the ion-ion structure factor and $n(k)$ is identified as the pseudo-electron density, or pseudo-atom (of valence Z). Thus electron-ion correlations can be described by convolving the pseudo-atom with the ionic correlations. The ionic correlations are themselves well described by a Percus-Yevick hard-sphere structure factor [3], while the pseudo-atom is described by a standard linear response formulation:

$$
n(k)=\chi_{1}(k) v^{p s}(k)
$$


where $\chi_{1}(k)$ is the well known linear response function; to approximate $\chi_{1}(k)$ we use a Local Density Approximation (LDA) local field factor [4]. The electron-ion interaction is modeled by a simple local one-parameter empty-core pseudopotential [0] i.e.: $v^{p s}(k)=$ $-\left(4 \pi e^{2} / k^{2}\right) \cos \left(k R_{c}\right)$, where $R_{c}$ is the core radius and the pseudo-potential goes through zero at $k_{0}=\pi / 2 R_{c}$. (We note that at this linear level, the effects of ionic averaging on the pseudo-atom are ignored [6]). Using the approximations above in (11), we compare our approach in Fig. 1 to the full ab-initio Car-Parrinello [7] calculations of de Wijs et al [8]. The correspondence is striking, especially when we note that the parameters $\eta$ and $R_{c}$ are a priori set by other physical properties (no fitting is necessary).

Besides a semi-quantitative description of electron-ion structure factors, this linear response theory now provides an important qualitative insight into the form of the electron-ion structure factors [9]. The pseudo-atom density, $n(k)$, is typically largest for smaller $k$ and thereafter rapidly declines for larger $k$, while the near classical ion-ion structure factor, $S_{I I}(k)$, follows an inverse behavior; it is small for small $k$. Together with the product form (11) this implies that the shape of the electron-ion structure factor, $S_{e I}(k)$, is determined primarily by the the position of the zero-crossing, $\bar{k}_{0}$, of $n(k)$ with respect to the first maximum, $k_{p}$, of $S_{I I}(k)$. If $\bar{k}_{0}<k_{p}$, then $S_{I I}(k)$ selects (or filters) the negative part of $n(k)$ and $S_{e I}(k)$ takes a form similar to that of Mg (Fig. 1 (a)). Conversely, if $\bar{k}_{0}>k_{p}$, then the ion-ion structure factor selects (or filters) the positive part of $n(k)$, and again, $S_{e I}(k)$ takes a form similar to that of $\mathrm{Bi}$ (Fig. 1 (1) (c)). Since $\chi_{1}(k)$ is positive definite, the zero-crossing in linear response occurs at $k_{0}$. The large slope of $n(k)$ near the zero-crossing then implies that non-linear corrections must have a small effect on the location of the zero-crossing, and this, together with the expected accuracy of linear response, implies that $\bar{k}_{0} \sim k_{0}$. For most metals, $k_{0}$ is just a little less than $2 k_{F}$, and the latter's ratio to $k_{p}$ is well known: for low valence $(Z \leq 2), 2 k_{F}<k_{p}$; for high valence $(Z \geq 3): 2 k_{F}>k_{p}$ [10]. This accounts in a straightforward way for the two separate forms found by deWijs et al $[\mathbb{8}]$ : For $\mathrm{Mg}, \bar{k}_{0}<k_{p}$ $(Z=2)$, which belongs to the low valence class of electron-ion structure factors. For $\mathrm{Bi}, \bar{k}_{0}>k_{p}(Z=5)$ and we may refer to this as the high valence class of electron-ion 
structure factors [11]. Generally ions of valence $Z \leq 2$ belong to the low valence class while ions with valence $Z>3$ belong to the high valence class. Ions with valence $Z=3$ typically belong to the high valence class also, although they may be characterized by a crossover form [6]. The analytical approach above can easily be extended by using the modern theory of classical liquids to obtain improved ion-ion structure factors [12], but to include second order contributions to the pseudo-atom $n(k)$ necessitates not only second order electron response, but also contributions from ion-ion triplet structure. The latter can be carried out with concepts from the theory of classical liquids [6].

\section{B. Proposed Experiments}

The principal features of electron-ion structure factors can be measured by exploiting the differences between the x-ray scattering structure factor, $S_{I I}^{X}(k)$, determined with a freeatom form factor, $f_{A}(k)$, and the structure factor, $S_{I I}^{N}(k)$, determined by neutron scattering [13,14]. As emphasized by Chihara [14 the x-ray structure factor for liquid metals will equal $S_{I I}^{N}(k)$ only when determined with an ionic form factor augmented by a pseudo-atom form factor; i.e. $f_{I}(k)+n(k)$, so that:

$$
\frac{S_{I I}^{X}(k)}{S_{I I}^{N}(k)}=\frac{\left|f_{I}(k)+n(k)\right|^{2}}{\left|f_{A}(k)\right|^{2}} .
$$

This effect is clearly expected to be largest for metals with larger ratios of valence to core electrons. Thus we predict a small effect for metals with smaller valence to core ratio such as $\mathrm{Na}$ or $\mathrm{K}$, a $2 \%$ difference at the 1 st peak of the structure for $\mathrm{Li}$ (ratio= $1: 2$ ) or $\mathrm{Al}$ $($ ratio $=3: 10)$, but by far the largest effect for Be (ratio $=1: 1$ ) where the difference at the principal peak of the structure factor could be as high as $7 \%$, well within experimental range. Another interesting candidate would be metallic Si (ratio $=4: 10$ ) since covalent effects still make themselves felt in the liquid state suggesting that experiments could reveal effects beyond linear response.

To date the experimental electron-ion structure factors and related pseudo-atoms show considerably more structure than indicated by theoretical predictions [15]. Significant ex- 
perimental challenges are faced in the accuracy resulting from subtraction of two sets of data obtained by quite different means, each with important (but different) systematic corrections; however the present approach suggests that the current differences between x-ray and neutron scattering should be reexamined (see also [14]). Using a pseudo-atom instead of the full free atom as a form factor can assist in comparing neutron and x-ray measurements and help unravel various systematic corrections applied. The advent of high precision x-ray and neutron sources currently coming on-line suggests that these proposed effects can be systematically explored.

\section{Non-linear response of an atom in an electron gas}

The evident (and long-standing) success of the linear response approximation for electron response [4], here demonstrated for electron-ion structure factors, calls for further investigation. The accuracy of linear response in a crystalline solid is commonly attributed to the fact that the structure dependent reciprocal lattice vectors are typically near the pseudopotential zero-crossing, $k_{0}$, with the associated inference that the net scattering is smaller than one would naively expect [4]. For liquids or other disordered systems such arguments are less appropriate. To examine the strength of linear response in the absence of ionic structure, we consider a simpler problem, namely the response of the interacting electron gas to a single ion, where the electron-ion interaction is modeled by a simple local one-parameter empty-core pseudopotential [5]. We will compare two routes to the induced density, $\rho^{\text {ind }}(k)$. The first follows from solving the Kohn-Sham equations [2] exactly (for the given pseudopotential) within the local density approximation (LDA), the second from the standard expansion of the response in powers of the perturbing (pseudo)potential, i.e.;

$$
\rho^{i n d}(k)=\chi_{1}(k) v^{p s}(k)+\sum_{\vec{k}_{1}} \sum_{\vec{k}_{2}} \chi_{2}\left(k, k_{1}, k_{2}\right) v^{p s}\left(k_{1}\right) v^{p s}\left(k_{2}\right)+\cdots
$$

Here the response functions, $\chi_{n}\left(k_{1} \ldots\right)$, are properties of the homogeneous interacting electron gas. In Fig. 2 we compare the explicit second order response with an LDA local field 
factor [6,16] to the full non-linear LDA response. Clearly the non-linear response is well characterized by the 2nd order term, implying that the success of linear response is not due to cancellation between higher order terms of opposite sign but instead that each successive term is individually small compared to the previous term in the expansion; the response series converges very rapidly, term by term.

The non-linear response is largest for atomic parameter $R_{c}=0$ (hydrogen), and decreases with a larger atomic-parameter, $R_{c}$, as might be physically anticipated. However as $R_{c}$ increases from zero, a noticeable secondary minimum occurs when the inverse atomic length, $k_{0}$, is equal to $2 k_{F}$. For the cases plotted in Fig. 2, the maximum in second order response at $k_{0} / 2 k_{F}=1$ (or $R_{c} / r_{s}=0.41$ ) is reduced by an entire order of magnitude when compared with the maximum in second order response calculated for hydrogen $\left(R_{c} / r_{s}=0\right)$, and is typically equal to the value at $3 R_{c} / r_{s}$. The physics behind this minimum is attributed to the following; the second order response function, $\chi_{2}\left(k, k_{1}, k_{2}\right)$, peaks when the summed arguments in (4) are close to $2 k_{F}$ [6]. If the pseudo-potential zero-crossing, $k_{0}$, is near the response peaks at $2 k_{F}$, a maximal cancellation or maximal destructive interference of the atomic and electronic length scales occurs, leading to a minimum in second order response. The ratio of the atomic and electronic length scales is set primarily by the volume energy terms in the total ground state energy, and is almost independent of structure [4]; $k_{0} / 2 k_{F}$ lies between 0.75 and 1 for most metals, and is therefore very close to the secondary minimum in the non-linear response.

As noted, the effect we discuss originates from an interference between intrinsic atomic and electronic length scales, but it also complements the argument given for crystalline solids alone, which stems from the confluence of an atomic and a structural length scale. The clear exception to these interference effects is again the singular case of a point-charge $\left(v^{p s}(k) \sim 4 \pi e^{2} / k^{2}\right)$, i.e. the case of hydrogen, which has no well-defined core-length scale, $k_{0}$, no oscillations in the potential and thus no interference effect in the higher order terms. In contrast to other systems, non-linear response terms are large term by term. In fact, the response series may not even formally converge and care must be taken when applying 
concepts derived from linear-response theory to hydrogen (it is not a simple material).

Finally we note that the second order response contribution is of the same order as the difference between first order response with or without local field corrections. In addition, the combined effects of exchange and correlation partially cancel between first and second order, implying that neglect of higher order response results in an over-estimation of the role of exchange and correlation, which, in turn, has important implications for the widespread application of linear response theory in the derivation of effective ion-ion potentials in (simple) metals.

\section{CONCLUSIONS}

The electron-ion structure factors of liquid simple-metals are well described by a sim-

ple linear response theory augmented by linear response for the electrons. This approach suggests two main classes of electron-ion correlation functions, one for high and one for low valence metals. Experimental advances in x-ray and neutron-scattering may be able to provide measurements of these electron-ion correlation functions, with liquid Be being the most promising candidate. A route to information on fundamental electron-ion interactions therefore becomes available through the fluid state. Finally, the well documented success of the linear response approximation for electrons stems in part from an interference effect between atomic and electronic length scales.

This work was supported by the NSF through the Cornell Center for Materials Research under Grant No. DMR96-32275. We especially thank Professor Karsten Jacobsen for making an LDA Kohn-Sham program available to us, and Dr David Muller for helpful suggestions. 


\section{REFERENCES}

[1] N.W. Ashcroft and D. Stroud, Solid State Physics 33, 1 (1978).

[2] W. Kohn and L.J. Sham, Phys. Rev. 140, A1133 (1965).

[3] N. W. Ashcroft and J. Lekner, Phys. Rev. 165, 83 (1966).

[4] J. Hafner, From Hamiltonians to Phase Diagrams, (Springer Verlag, Berlin, (1987)).

[5] N.W. Ashcroft, Phys. Lett. 23, 48 (1966).

[6] A.A. Louis, Quantum Dissipation from Phonons; Metallic Hydrogen; Electron-Ion Mixtures, PhD Thesis, Cornell University (1997)

[7] R. Car and M. Parrinello, Phys. Rev. Lett. 55 , 2471 (1985).

[8] G. A. de Wijs, B. Pastore, A. Selloni, and W. van der Lugt, Phys. Rev. Lett. 75, 4480 (1995).

[9] A.A. Louis and N.W. Ashcroft, Phys. Rev. Lett. 81, 4456 (1998).

[10] J. M. Ziman, Principles of the Theory of Solids, 2nd Ed, (Cambridge University Press, Cambridge (1972)), p 227.

[11] In their interesting paper de Wijs et. al. [8] suggest that the difference between Mg and $\mathrm{Bi}$ electron-ion structure factors is partially due to the nearly free electron (NFE) bonding of $l$-Mg as against the remnant covalency of $l$-Bi. However a high valence NFE metal such as $l$-Pb should have the same form of $S_{e I}(k)$ as $l$-Bi.

[12] S. Cusack, N.H. March, M. Parrinello and M. P. Tosi, J. Phys F: Met. Phys. 6, 749 (1976). K. Hoshino and M. Watabe, J. Phys. Soc. Japan 61, 1663 (1992).

[13] P.A. Egelstaff, N.H. March and N.C. McGill, Can. J. Phys. 52, 1651 (1974).

[14] J. Chihara, J. Phys. F: Met. Phys. 17, 295 (1987). See also J.A. Anta, B.J. Besson, and P.A. Madden Phys. Rev. B. 58, 6124 (1998). 
[15] S. Takeda, S. Tamaki and Y. Waseda, J. Phys. Soc. Japan, 542552 (1985); S. Takeda, S. Harada, S. Tamaki and Y. Waseda, J. Phys. Soc. Japan, 55184 (1986); ibid 553437 (1986), ibid, 58, 3999 (1989), ibid 60, 2241 (1991), ibid 63 1794, (1994).

[16] P. Lloyd and C. Sholl J. Phys. C 1, 1620 (1968). 


\section{FIGURE CAPTIONS}

\section{FIGURE 1}

The electron-ion structure factors $S_{e I}(k)$ and related electron-ion correlation functions $g_{e i}(r)$ for $\mathrm{Mg}$ and $\mathrm{Bi}$ : Car-Parrinello results of de Wijs et al [8] (solid line) vs. the simple linear-response approach augmented by a hard-sphere approximation (dashed line). Panel (a) shows $S_{e I}(k)$ and panel (b) shows $g_{e I}(r)$ for liquid $\mathrm{Mg}$. Panel (c) shows $S_{e I}(k)$ and panel $(\mathrm{d})$ shows $g_{e I}(r)$ for liquid Bi. For $\mathrm{Mg}$ the parameters (taken from the literature) are: $r_{s}=2.66 a_{0}, R_{c}=1.31 a_{0}$ and for Bi the parameters (taken from the literature) are: $r_{s}=2.25 a_{0}$ and $R_{c}=1.15 a_{0}$. Both have a hard-sphere parameter, $\eta=0.46$. (note that for the $g_{e I}(r)$ the region inside the core radius is not physically significant.)

\section{FIGURE 2}

A comparison of full non-linear LDA response $\left[\rho(k)-\rho^{(1)}(k)\right]$ (solid line) to second order LDA response (dashed line) for an empty core pseudo-potential with $R_{c}=1.5 a_{0}$ embedded in an electron gas with density parameter, $r_{s}=3 a_{0}$. For the scale, compare this to the full response with the limit $\rho(k \rightarrow 0)=1$. The higher order response is of the order of a few $\%$ of the full response and in turn, the second order response captures almost all the non-linear response. (The small difference at $k \rightarrow 0$ is a numerical artifact stemming from the use of a large but finite real-space cut-off radius in the Kohn-Sham procedure.) In the insert is plotted the maximum of the 2 nd order response vs. $R_{c} / r_{s}$ for $r_{s}=2 a_{0}$ (dotted), $r_{s}=3 a_{0}($ solid) and $r_{s}=5 a_{0}$ (dashed). Note especially the minimum at $R_{c} / r_{s}=0.41$ which corresponds to $k_{0}=2 k_{F}$. It is reduced by an order of magnitude from the value at $R_{c}=0$ (hydrogen) and is traced to an interference between atomic and electronic length-scales. 


\section{FIGURES}
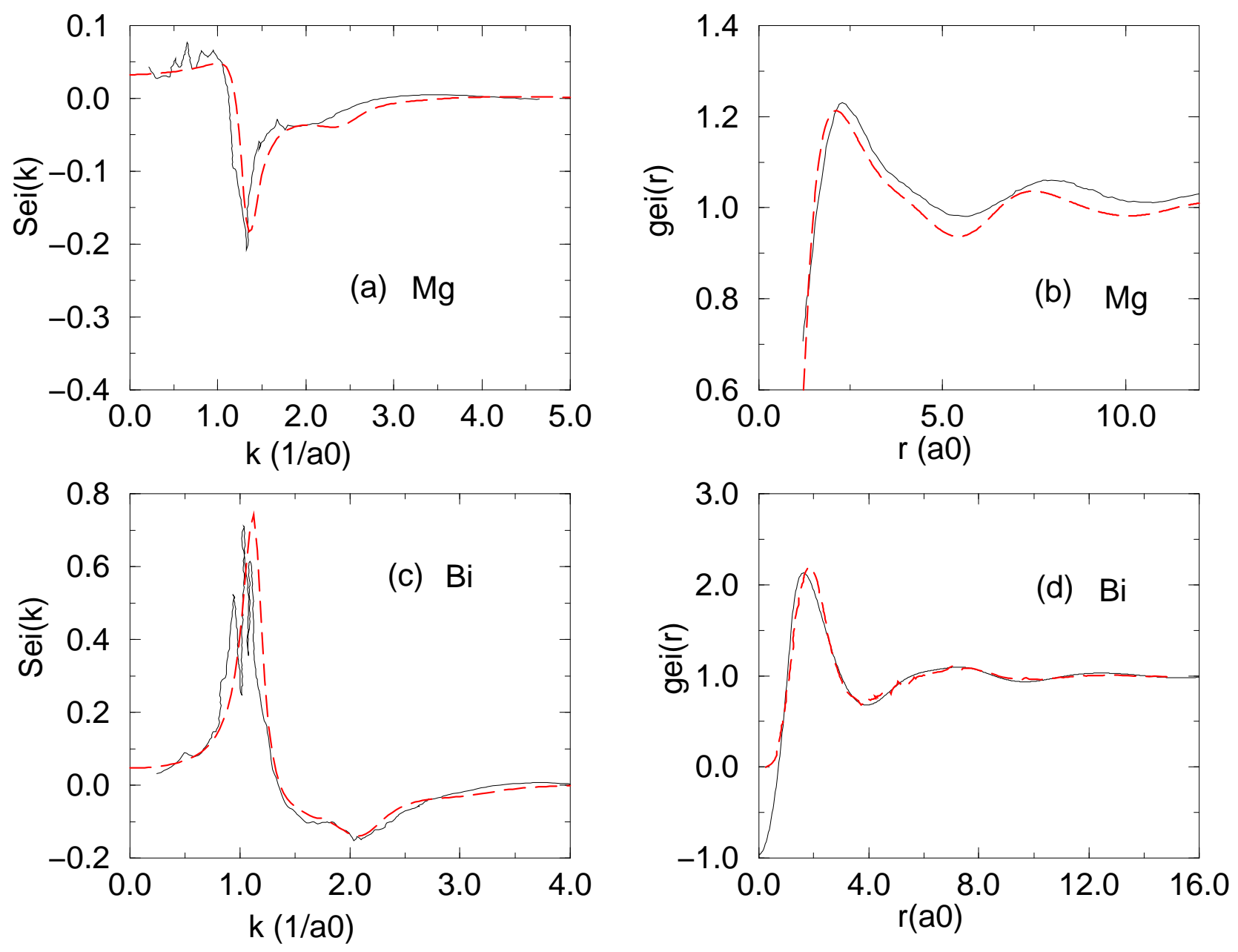

FIG. 1. 


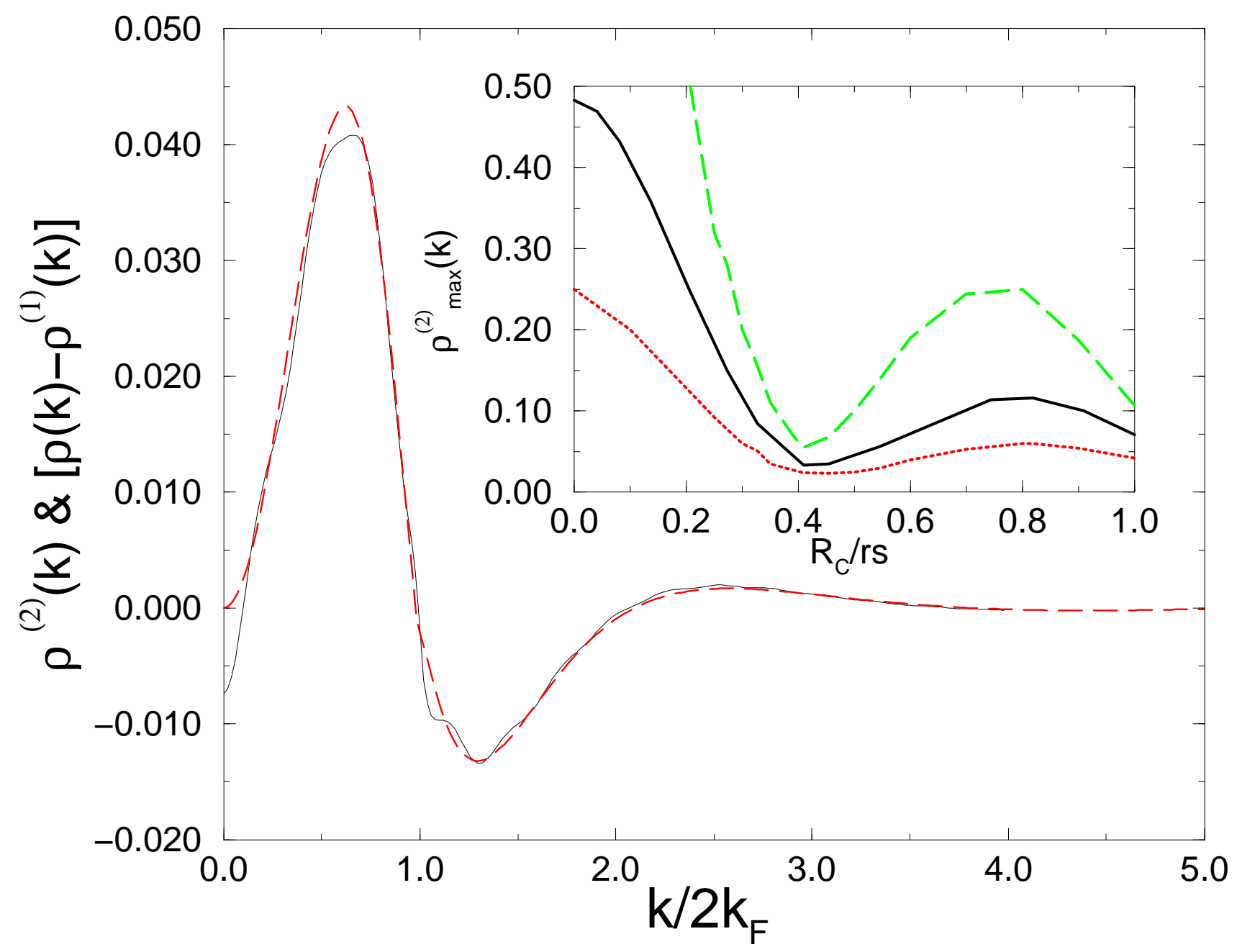

FIG. 2. 